\title{
BIOPSIES TESTICULAIRES PERCUTANEES DANS L'EXPLORATION DES INFERTILITES MASCULINES
}

\author{
D. Delavierre*, G. Fournier*, A. Volant**, P. Philippe*, Ph. Mangin* \\ * Service d'Urologie CHU de Brest \\ **Service d'Anatomo-pathologie CHU de Brest
}

\section{PERCUTANEOUS TESTICULAR BIOPSY USING AN AUTOMATIC GUN IN THE EXPLORATION OF MALE INFERTILITY. Between February 1989 and March 1991, eight patients (mean age 32 years) presenting with azoospermia or severe oligozoospermia underwent a testicu- lar biopsy to investigate their secretory or excretory origin. A bilaterial testicular biop- sy was performed percutaneously using an depuis la réalisation des dosages hormonaux plasmatiques cette technique reste néanmoins la seule permettant d'apprécier directement la quali- té de la spermatogenèse. Nous rapportons notre expérience de la biopsie testiculaire percutanée au pistolet automatique au cours de l'exploration des stérilités masculines. \\ MATERIELS ET METHODES Population} automatic biopsy gun with a Tru-cut 14 gauge needle. General anaesthesia was used for seven patients although one man had a painless biopsy following spermatic cord anaesthesia. Satisfactory specimens were obtained in seven patients (more than 30 spermatogenic tubules visible in each biopsy), and the differential diagnosis of excretory (three cases) or secretory origin (four cases) of the infertility was possible. In six patients a testicular ultrasonographic examination was performed after the biopsy. Results were normal in five men while the sixth showed a haematocoele, although the fluid was removed percutaneously. In 7/8 patients no haemorrhagic or infectious complications were noted. To investigate possible immediate complications of this type of procedure, 25 patients presenting with prostatic carcinoma underwent percutaneous testicular biopsy just before their orchidectomy. In all patients a haematic infiltration of the testicular parenchyma along the needle's path was observed. Among these 25 men, eight biopsies were performed under local spermatic cord anaesthesia : the procedure was painless in six cases but slightly painful in two. Consequently, percunateous testicular biopsy using an automatic gun is an interesting option compared to the traditional open surgical approach used to investigate spermatogenesis in certain types of male sterility. Furthermore, this procedure can be performed under local anaesthesia in the consulting room, making this alternative approach even more attractive. Key words : Testicular biopsy, automatic biopsy gun. Andrologie, 1992, 2 : 8-9.
Si l'intérêt de la biopsie testiculaire a diminué

Entre Février 1989 et Mars 19918 hommes, agés de 27 à 40 ans (moyenne 32 ans), explorés pour azoospermie ou oligospermie sévère, ont eu une biopsie testiculaire percutanée afin de connaitre l'origine sécrétoire ou excrétoire de l'anomalie. Cette biopsie a été réalisée sous anesthésie générale chez 7 patients et locale chez le dernier patient.

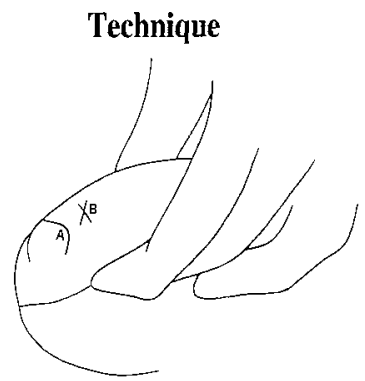

Figure 1 : Repérages préalables ì la ponction. A Projection de la queue épididymaire sur la peau scrotale ; B Point de ponction

Le matériel comporte une aiguille de type tru-cut 14 gauge et un pistolet automatique. L'anesthésie locale nécessite une seringue de $20 \mathrm{cc}$ de xylocaïne munie d'une aiguille de type sous-cutanée 25 gauge. Cette anesthésie s'effectue par infiltration des cordons spermatiques dans leur partie scrotale, puis de la peau dans la région où pénètrera l'aiguille de ponction.

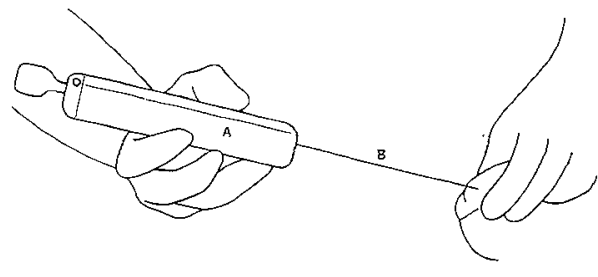

Figure 2 : Matériel utilisé. A Pistolet automatique ; B Aiguille TruCut 14 gauge
Une main saisit et maintient le testicule et l'épididyme. L'autre main repère la queue épididymaire. Une moucheture est réalisée au bistouri à environ $1 \mathrm{~cm}$ de la queue épididymaire.

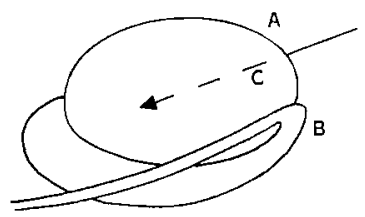

Figure 3 : Trajet de la ponction. A Pôle inférieur du testicule ; B Queue de l'épididyme; C Axe.

L'aiguille est introduite dans l'incision puis dirigée obliquement du pôle inférieur vers le pôle supérieur du testicule. En effet la biopsie s'effectue dans l'axe longitudinal du testicule, en évitant l'épididyme. L'aiguille est déclenchée et la biopsie est ainsi réalisée. Après ablation de l'aiguille le point de ponction est comprimé.

La biopsie s'effectue selon la même technique des 2 côtés. L'échoguidage de la ponction est possible mais complique la manipulation sans apporter d'avantages par rapport au repérage manuel.

\section{RESULTATS}

Le patient chez qui la biopsie a été réalisée sous anesthésie locale n'a ressenti aucune douleur.

Les prélèvements ont été suffisants chez 7 patients (plus de 30 tubes séminifères dans chaque biopsie) pour diagnostiquer l'origine excrétoire ( 3 cas) ou sécrétoire (4 cas) de l'infertilité.

Une échographie testiculaire a été réalisée 24 heures après la biopsie chez 6 patients. Normale 5 fois, elle a mis en évidence une hématocèle dans un cas. Chez ce dernier patient une évacuation de l'hématocèle a été effectuée par simple ponction.

Chez 7 patients aucune complication hémorragique ou infectieuse n'est apparue.

\section{Discussion}

Les indications de la biopsie testiculaire percutanée sont identiques à celles de la biopsie chirurgicale classique ( c'est à dire l'exploration des 
azoospermies et des oligospermies sévères à FSH normale[2,4] ). Elle est contre-indiquée lors de troubles de la coagulation.

Le matériel histologique obtenu est de bonne qualité $(1,3,4)$. C'est notamment la conclusion de Cohen qui a comparé le matériel obtenu avec une biopsie testiculaire percutanée et celui obtenu avec une biopsie chirurgicale classique chez 10 patients (1).

Les risques principaux de ce geste sont:

- Ia plaie épididymaire (facilement évitée grâce au repérage manuel de la queue épididymaire et à la direction donnée à l'aiguille)

- l'hématome testiculaire

Dans un but expérimental, afin de préciser les conséquences immédiates des biopsies sur le testicule et ses annexes, nous avons également réalisé celles-ci immédiatement avant castration chez 25 patients atteints de cancer de prostate. 8 biopsies ont été effectuées sous anesthésie locale du cordon spermatique, sans douleur 6 fois, avec une douleur modérée 2 fois. Le point de pénétra- tion de l'aiguille au niveau de l'albuginée a toujours été repéré au pole inférieur du testicule près de la queue épididymaire. Une infiltration hématique du parenchyme testiculaire sur le trajet de l'aiguille de ponction a été observée chez tous les patients.

\section{Conclusion}

La biopsie testiculaire percutanée au pistolet automatique est un geste simple, rapide et peu agressif (4). Elle peut s'effectuer sous anesthésie locale $(3,4)$ et permet d'obtenir le plus souvent un matériel histologique de bonne qualité. Elle constitue donc une alternative intéressante à la biopsie chirurgicale classique.

\section{REFERENCES}

1 - Cohen M.S., Ftye S., Warner R.S., Leiter E. : Testicular needle biopsy in diagnosis of intertility Urology 1984, 24 : 439-442.

2 - Marmor D. : La biopsie testiculaire, in : Andrologie Tome 1, G. Arvis ed., Maloine, Paris 1987: 237-241.
3 - Netto N.R. : Needle Method of testicular Biopsy. Int. Surg. 1974, $59: 172$.

4 - Rajfer J., Binder S. : Use of biopsy gun for transcuteneous testicular biopsies J. Urol. 1989, 142 : 1021-1022.

RESUME : Une biopsie testiculaire percutanée bilatérale a été réalisée chez 8 hommes explorés pour azoospermie ou oligospermie sévère. Celle-ci s'effectue au pistolet automatique à l'aiguille 14 gauge de type Tru-cut. Les prélèvements ont été suffisants par le diagnostic chez 7 patient. Un patient a présenté une hématócèle unilatérale traitée par simple ponction. Ce geste simple, rapide et peu agressif peut s'effectuer sous anesthésie locale et constitue une alternative intéressante à la biopsie chirurgicale classique. Mots clés : Biopsie testiculaire, Infertilité masculine, Pistolet automatique à biopsie. Andrologie, 1992, $2: 8-9$.

\section{INSTRUCTIONS AUX AUTEURS}

Les textes scientifiques publiés dans Andrologie doivent être constitués comme suit, et adressés en quatre exemplaires au rédacteur en chef:

1. Page de garde comportant titre, nom(s), prénom(s) et adresse(s) de(s) institution(s) de(s) auteur(s).

2. Page suivante comportant un titre anglais, un résumé anglais plus long et détaillé que le résumé français, mais ne devant pas dépasser 30 lignes dactylographiées double interligne, et une liste de 2 à 6 mots clés en anglais.

3. A partir de la page suivante, texte dactylographié en double interligne sur un seul côté de la page, et seion un format normalisé : 27 lignes de 60 caractères et espaces par ligne. Chaque fois que possible, la présentation doit suivre un plan classique, c'est à dire : Introduction, Matériel et méthodes, Résultats, Discussion, Références. Le texte doit être le plus concis possible.

4. Les références sont à présenter sous le titre "REFERENCES", et par ordre alphabétique. Elles soivent être appelées dans le texte par leur numéro. Leur nombre doit être limité à 10 au maximum pour 4 pages de texte (20 pour 8 pages, 30 pour 12 pages, sauf cas particulier de revue générale). Suivre le modèle ci-après :

1 - Auroux M, Dulioust E. Cyclophosphamide in the male rat : Behavioral effects in the adults in artificial insemination. In : David G, Price WS eds. Human artificial insemination and semen preservation. New York, Plenum Press, $1980: 197-210$.

2 - Schwartz D, Mayaux MJ. Mode of evaluation of results in artificial insemination. In : David G, Price WS eds. Human artificial insemination and semen preservation. New York, Plenum Press, 1980 : 197-210.

Lorsqu'il y a plus de six auteurs, ne citer que les 3 preniers, suivis de la mention "et al".

5. Sur la page suivant les références, placer un résumé en français court, maximum 10 lignes dactylographiées double interligne, suivi d'une liste de 2 à 6 mots clés en français.

6. Les tableaux et figures doivent être présentés à part, 1 par page. Les légendes des différentes figures doivent être dactylographiées sur une page séparée. Le numéro des figures doit être inscrit au dos de chacune, au crayon de bois. Les photos en noir et blanc peuvent être reproduites, mais pas les photos et illustrations en couleur. 